\title{
Sickle-shaped transposition flap oriented along relaxed skin tension lines for lower eyelid reconstruction
}

\author{
Tae Hyeon Lee, Hyun Joon Seo, \\ Seong Oh Park
}

Department of Plastic and Reconstructive Surgery, Hanyang University College of Medicine, Seoul, Korea
Background The reconstruction of defects in the lower eyelid region is prone to complications such as ectropion and epiphora. This area is also aesthetically important; therefore, operations should be carried out with caution. We introduce a simple and easy surgical approach for the repair of small to moderate-sized lower eyelid defects. Methods A retrospective chart review was performed for all patients who underwent lower eyelid defect reconstruction using a sickle-shaped transposition flap in 2018 or 2019. Photographs were taken at each visit, and the Vancouver Scar Scale was used to evaluate residual marks every 3 months for 1 year. Patients reported their subjective satisfaction levels on a visual analogue scale that ranged from 0 to 10.

Results A total of nine patients were included. No flap necrosis, ectropion, epiphora, or other complications were observed. Among the eight patients with skin cancer, no recurrence was noted during the follow-up period. The mean Vancouver Scar Scale scores were $5.50 \pm 0.99$ at 3 months postoperatively, $4.61 \pm 0.85$ at 6 months postoperatively, $3.05 \pm 0.80$ at 9 months postoperatively, and $1.11 \pm 0.58$ at 12 months postoperatively. The mean overall patient satisfaction rating was $9.11 \pm 0.78$ (as rated on the visual analogue scale).

Conclusions In the reconstruction of lower eyelid defects, the sickle-shaped transposition flap could be a simple, fast, and aesthetically favorable surgical option.

Keywords Surgical flaps / Reconstructive surgical procedures / Ectropion

\section{INTRODUCTION}

Facial defects have numerous causes, including skin cancer, trauma, and congenital malformations. Various methods exist to address these issues, with the chosen approach varying depending on the defect size and position [1-6]. Defects near vital structures such as the periorbital, perioral, and perialar areas must be reconstructed without any deformities, as deformities in these regions can

Received: Jun 11, 2020 Revised: Jul 28, 2020 Accepted: Jul 29, 2020 Correspondence: Seong Oh Park Department of Plastic and Reconstructive Surgery, Hanyang University College of Medicine, 222-1 Wangsimni-ro, Seongdong-gu, Seoul 04763, Korea

Tel: +82-2-2290-8567, Fax: +82-2-2295-7671, E-mail: psopark950@gmail.com

Copyright @ 2020 The Korean Society for Aesthetic Plastic Surgery.

This is an Open Access article distributed under the terms of the Creative Commons Attribution Non-Commercial License (https://creativecommons.org/licenses/by-nc/4.0/) which permits unrestricted non-commercial use, distribution, and reproduction in any medium, provided the original work is properly cited. $\quad$ www.e-aaps.org cause functional and aesthetic problems [3]. The reconstruction of lower eyelid defects is prone to complications, such as ectropion and epiphora, and therefore requires caution [3]. Defects can be easily covered with grafts, but depression deformities can arise, and color differences with surrounding tissues may be present [6]. Rhomboid flaps are geometrically random pattern-designed fullthickness cutaneous transposition flaps. These flaps can cover defects with tissues of similar thickness and color, making them common choices for facial reconstruction [7]. However, rhomboid flaps also have disadvantages, such as the potential for tension, additional scarring, or dog-ear deformity [7]. Advancement flaps are also a popular reconstructive option, but they require long additional incisions for tension-free closure.

In this report, we introduce and analyze a sickle-shaped flap (a transposition flap) as a simple reconstructive method for the repair of small to moderate-sized lower eyelid defects. 


\section{METHODS}

\section{Patients and ethical approval}

This investigation was reviewed and approved by the Institutional Review Board of Hanyang University Seoul Hospital (IRB No. 202006-018). A retrospective chart review was performed for all patients who underwent wide excision and reconstruction after skin cancer or trauma in 2018 or 2019. Of this larger group, patients who underwent reconstruction using a sickle-shaped local flap were included in the study. Tumor locations were limited to the medial canthal, lower eyelid, and lateral nose areas, presented as zones 1 and 2 in a previous article [8]. Patients with insufficient medical records or with a follow-up period of less than 1 year were excluded.

\section{Surgical method}

After wide excision of the skin cancer or debridement, the defect was measured. The width and length of the defect were the primary indicators for the flap design. The flap base was designed to be approximately twice the defect's width. The incision was started at the end of the defect and extended along the relaxed skin tension lines (RSTLs). The incision length was approximately 1.5 times the defect length. The defect was closed with the migrated flap, and dissection was then performed around the donor site. If the flap became subject to tension during donor site closure, non-absorbable sutures were placed between the deep structure and the lower flap to prevent traction of the recipient site (Fig. 1A). If the flap tip was too narrow or short to cover the defect, the tip was moved to a more distal point. This modification enabled coverage of the defect without tension (Fig. 1B).

\section{Patient evaluation}

Follow-up evaluations were performed every 3 months for 1 year and thereafter twice a year for an additional 2 years. Photographs were taken at every visit, and the Vancouver Scar Scale was used to evaluate residual marks every 3 months until 1 year postoperatively. Analysis of the photographs was performed by one of whom was the operator (SOP) and the other a third-year resident (THL). Patients reported their subjective satisfaction using a visual analogue scale ranging from 0 to 10 for the subcategories of pain, dry eye, erythema, edema, and ectropion, as well as their overall satisfaction at 1 year postoperatively.

\section{RESULTS}

A total of nine patients were included. The mean follow-up period was 18.39 months (range, 13.73-26.03 months). The defect etiologies were basal cell carcinoma $(7 / 9,77.8 \%)$, squamous cell carcinoma $(1 / 9,11.1 \%)$, and trauma $(1 / 9,11.1 \%)$. The defects were measured along the long and short axes. The mean length of the long axis was $15.27 \mathrm{~mm}$ (range, $11.58-19.29 \mathrm{~mm}$ ) and that of the short axis was $8.46 \mathrm{~mm}$ (range, $6.18-10.72 \mathrm{~mm}$ ). The majority of the defects were located on the lower eyelid $(6 / 9,66.7 \%)$, followed by the medial canthal area $(2 / 9,22.2 \%)$ and the lateral nose $(1 / 9,11.1 \%)$ (Table 1).

No flap necrosis, ectropion, epiphora, or other complications were observed. Among the eight patients with skin cancer, no recurrence was noted during the follow-up period. The mean Vancouver Scar Scale score was $5.50 \pm 0.99$ after 3 months, $4.61 \pm 0.85$
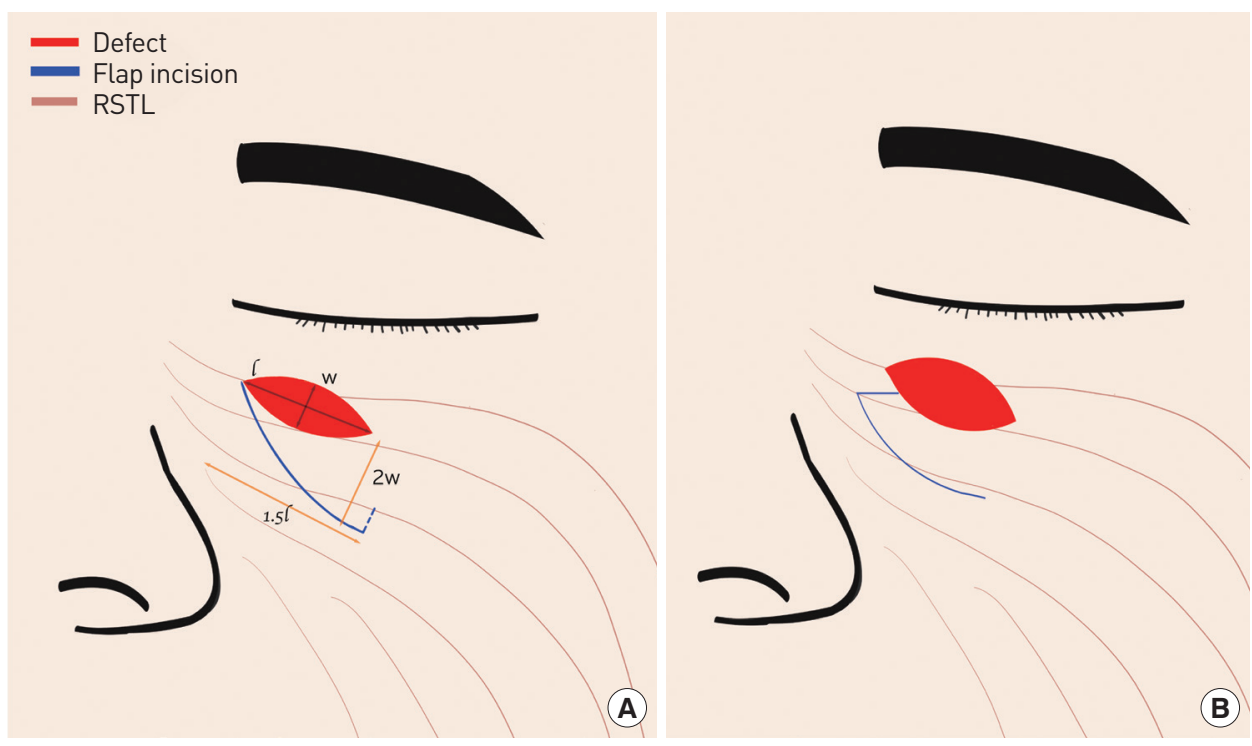

Fig. 1. Schematic illustration of the surgical method. The flap base is approximately twice the defect width. The flap length is approximately 1.5 times that of the defect. (A) When more tension-free rotation is required, a small back-cut incision can be added (blue dotted line). (B) Modification: if the flap tip is too narrow to fill the defect or too short to cover it, the flap tip should be moved to a more distal point. RSTL, relaxed skin tension line. 
Table 1. Patient characteristics

\begin{tabular}{lc}
\hline Variable & Value \\
\hline Sex & $3(33.3)$ \\
Male & $6(66.7)$ \\
Female & $68.67(39-86)$ \\
Age lyr) & $18.39(13.73-26.03)$ \\
Follow-up period (mo) & \\
Etiology & $7(77.8)$ \\
BCC & $1(11.1)$ \\
SCC & $1(11.1)$ \\
Trauma & $15.27(11.58-19.29)$ \\
Defect size (mm) & $8.46(6.18-10.72)$ \\
Long axis & \\
Short axis & $6(66.7)$ \\
Defect location & $2(22.2)$ \\
Lower eyelid & $1(11.1)$ \\
Medial canthal area & \\
Lateral nose &
\end{tabular}

Values are presented as number (\%) or mean (range).

BCC, basal cell carcinoma; SCC, squamous cell carcinoma.

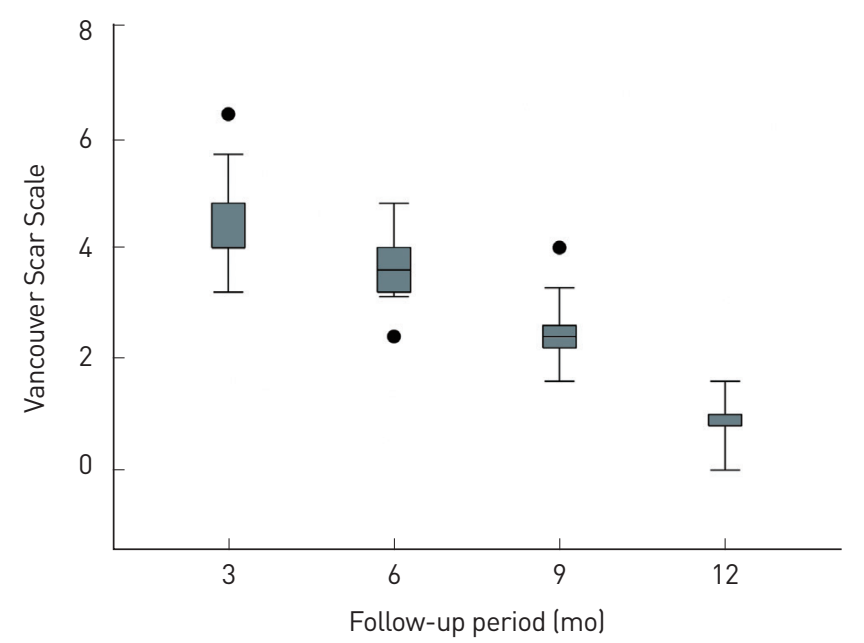

Fig. 2. Vancouver Scar Scale scores throughout the follow-up period.

after 6 months, $3.05 \pm 0.80$ after 9 months, and $1.11 \pm 0.58$ after 1 year (Fig. 2). The mean overall patient satisfaction rating was $9.11 \pm 0.78$ (as rated on the visual analogue scale) (Table 2).

\section{Case 1}

A 78-year-old woman was diagnosed with basal cell carcinoma of the right lower eyelid. Preoperatively, a punch biopsy was used to confirm a diagnosis of basal cell carcinoma, and the patient was referred to the plastic surgery department for wide excision. Excision was performed under local anesthesia, resulting in a $1.5 \times 0.6-\mathrm{cm}$ oblique defect in the lower eyelid region. The incision was de-
Table 2. Outcomes

\begin{tabular}{lc}
\hline Variable & Value \\
\hline Flap necrosis & $0 / 9$ \\
Ectropion & $0 / 9$ \\
Epiphora & $0 / 9$ \\
Other complications & $0 / 9$ \\
Tumor recurrence & $0 / 8$ \\
Mean Vancouver Scar Scale rating & \\
3 Months & $5.50 \pm 0.99$ \\
6 Months & $4.61 \pm 0.85$ \\
9 Months & $3.05 \pm 0.80$ \\
12 Months & $1.11 \pm 0.58$ \\
Mean patient satisfaction & \\
VAS score & $9.11 \pm 0.78$ \\
\hline
\end{tabular}

Values are presented as number/number or mean \pm SD.

VAS, visual analogue scale.

signed in an RSTL-oriented direction with a length of $2.5 \mathrm{~cm}$, which was approximately 1.7 times the defect length. The flap base was $1.2 \mathrm{~cm}$, approximately twice the defect width. Inferiorly, the lower lid was subject to slight tension, and temporary tarsorrhaphy was placed to prevent ectropion and removed on postoperative day 5. To prevent postoperative hematoma, a small Penrose drain was inserted; this was removed the day after surgery. At 12 months postoperatively, the Vancouver Scar Scale score was 2, and patient satisfaction as rated on the visual analogue scale was 9 (Fig. 3).

\section{Case 2}

A 45-year-old man was diagnosed with basal cell carcinoma of the left lower eyelid. Wide excision was performed under local anesthesia, resulting in a $1.3 \times 1-\mathrm{cm}$ defect on the medial side of the left lower eyelid. The defect was circular, with its longitudinal axis parallel to the RSTL. In such cases, a conventionally designed flap would have too narrow a tip, providing insufficient coverage of the defect. To address this concern, sufficient tissue can be secured using a modified method that moves the flap tip. This technique was utilized in this case, and no postoperative complications occurred. At 12 months postoperatively, the patient's Vancouver Scar Scale score was 1 , and patient satisfaction was 10 as rated on the visual analogue scale (Fig. 4).

\section{DISCUSSION}

The face includes various sensory organs, the improper functioning of which impairs appropriate response to stimuli and decreases quality of life. The eyelid is the site of $5 \%$ to $10 \%$ of all skin cancers [9]. Complete remission of skin cancer typically requires wide excision, a method that creates large defects in the eyelid region [10]. 

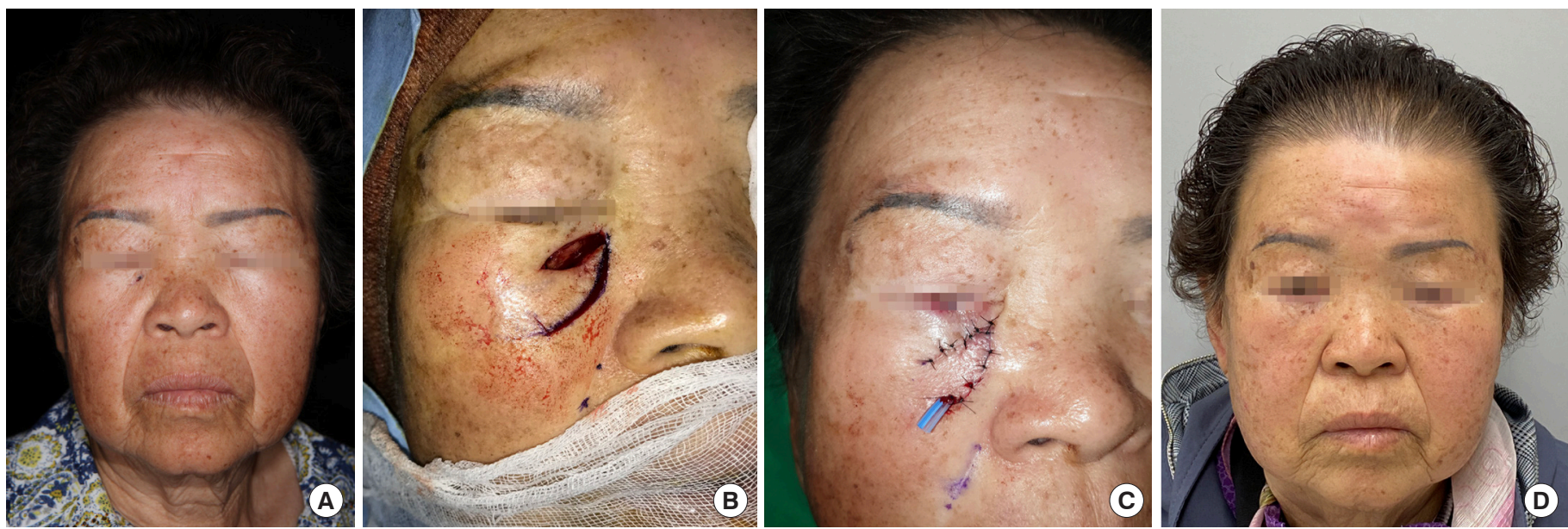

Fig. 3. A 78-year-old woman diagnosed with basal cell carcinoma of the right lower eyelid. (A) Preoperative photograph. (B) Intraoperative photograph after wide excision and elevation of sickle-shaped flap. (C) Immediate postoperative photograph. (D) Twelve months postoperative photograph.
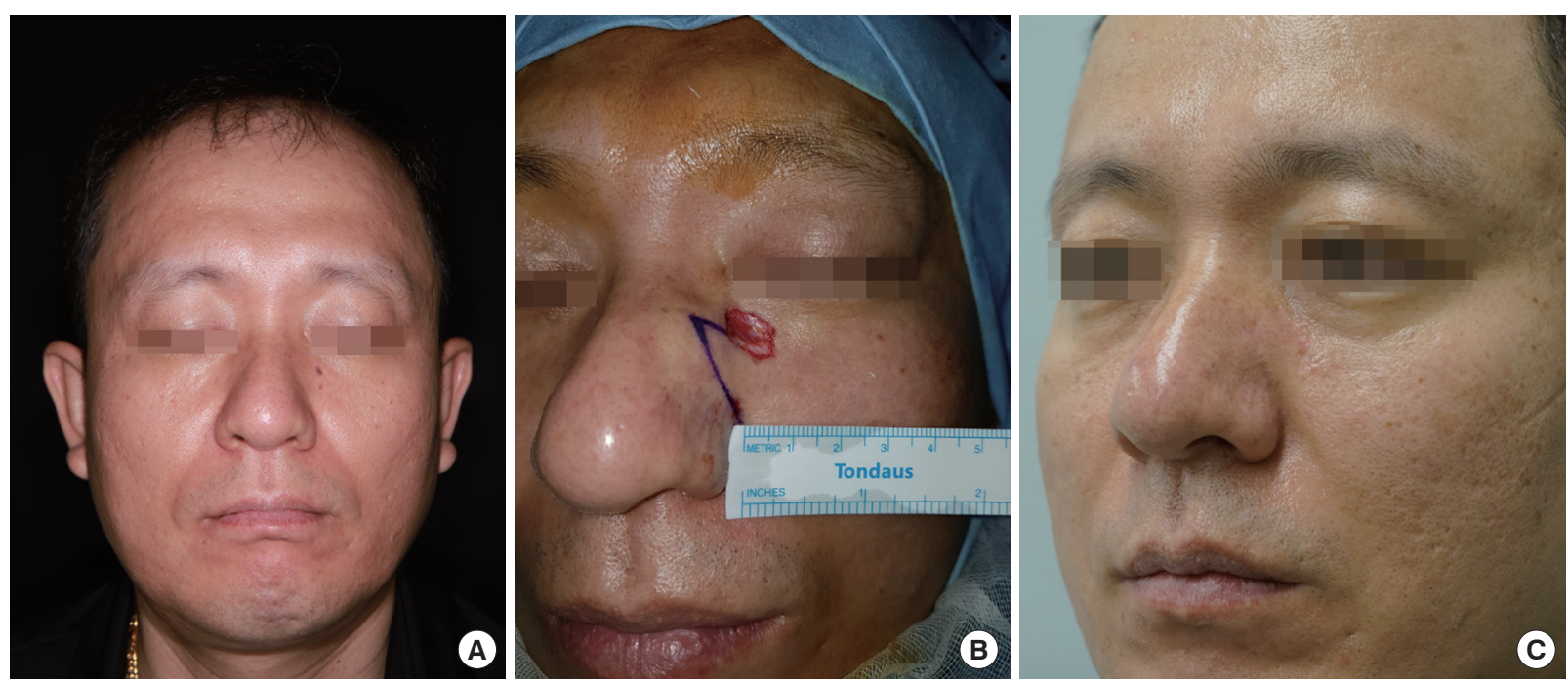

Fig. 4. A 45-year-old man diagnosed with basal cell carcinoma of the left lower eyelid. (A) Preoperative photograph. (B) Intraoperative photograph after wide excision and design of modified sickle-shaped flap. (C) 12 months postoperative photograph.

Defects of the facial skin and soft tissue are related to the physiological and anatomical functions of the sensory organs, and as such, appropriate coverage methods must preserve functionality. Managing skin defects around the lower eyelid requires particular attention due to the possibility of complications such as eyelid malpositioning, epiphora, lid edema, and hypertrophic scarring [3]. Ectropion refers to the exposure of the conjunctiva due to eversion of the eyelid margin. It is the most common eyelid malposition [11] and can seriously impact daily life $[12,13]$. In patients with this condition, foreign bodies enter and irritate the eye with greater frequency, causing redness, infection, and keratitis; in severe cases, corneal ulceration and infectious keratitis can develop. If ulceration and infection are not treated, corneal perforation or blindness can result [14]. To address defects in this region, multiple surgical coverage methods exist, with more in development. The aim of these techniques is to preserve a natural aesthetic appearance and to minimize scarring, while maintaining the eyelid's complex anatomy and function $[3,4,10,15]$.

The use of a sickle-shaped flap to cover lower eyelid defects has several advantages over more traditional approaches. When a local flap is used instead of primary closure, an additional scar from the flap elevation inevitably remains. Since the long axis of the sickleshaped flap runs parallel to the RSTLs, this additional scar is formed more naturally and has better cosmetic results. In addition, the tension is lower, decreasing the risk of eyelid malposition complications. A final benefit is that this method is very simple and can therefore be 


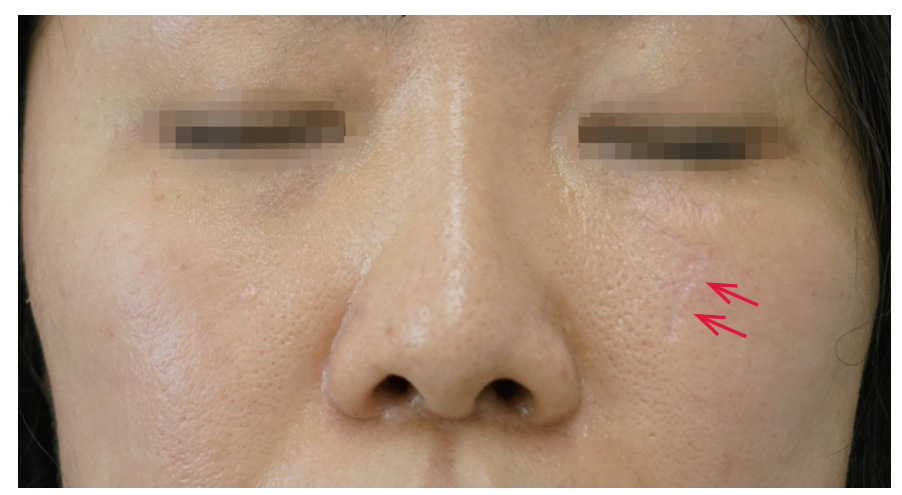

Fig. 5. Example of a Limberg flap that did not match the relaxed skin tension line (RSTL) direction. Incisions that do not align with the RSTLs can leave visible scarring (red arrows).

easily and quickly completed, even under local anesthesia.

In this study, lower eyelid region defects were analyzed in nine patients who underwent sickle-shaped local flap reconstruction oriented along the RSTLs. All nine sickle-shaped local flaps were taken well without acute complications such as necrosis, and no instances of ectropion or epiphora were encountered during the follow-up period.

The sickle-shaped flap has some disadvantages. First, the scars resulting from the use of such flaps are longer than those associated with Limberg or Dufourmentel flaps used to cover similar-sized defects. However, a shorter scar is not always cosmetically preferable. Elevating flaps in a direction that is inconsistent with the RSTLs may result in shorter scars, but these scars will likely be more prominent (Fig. 5). Sickle-shaped flaps may yield longer scars, but because the scars run parallel to the RSTLs, they remain pliable and flat, with a color similar to that of normal skin. In some cases, therefore, the aesthetic results are better with the sickle-shaped flap.

Another disadvantage is the difficulty in applying this technique across all areas. In addition to the RSTL-defect relationship, it is necessary to consider the location of surrounding landmark structures, which can complicate the application of this technique. Also, it may be difficult to employ sickle-shaped flaps in the repair of circular defects. In such cases, with due consideration of the surrounding landmarks and RSTL, one end of the defect can be cut to form an oval shape.

The present study has several limitations. Since this was a retrospective review, we could not control all of the variables that influenced the outcome. The number of cases was small because we limited the included patients based on the defect area (medial canthal area, lower eyelid, and lateral nose). In addition, we did not objectively compare this surgical method to others, and therefore the benefits of this approach could not be accurately quantified.

In the reconstruction of lower eyelid defects, the sickle-shaped transposition flap could be a simple, fast, and aesthetically favorable surgical option.

\section{NOTES}

\section{Conflict of interest}

No potential conflict of interest relevant to this article was reported.

\section{Ethical approval}

The study was approved by the Institutional Review Board of Hanyang University Seoul Hospital (IRB No. 2020-06-018) and performed in accordance with the principles of the Declaration of Helsinki.

\section{Patient consent}

The patients provided written informed consent for the publication and the use of their images.

\section{ORCID}

Tae Hyeon Lee

Hyun Joon Seo

Seong Oh Park

https://orcid.org/0000-0002-2806-3885

https://orcid.org/0000-0003-0977-2579

https://orcid.org/0000-0001-8990-0635

\section{REFERENCES}

1. Johnson AR, Egeler SA, Wu WW, et al. Facial reconstruction after Mohs surgery: a critical review of defects involving the cheek, forehead, and perioral region. J Craniofac Surg 2019;30:400-7.

2. Pepper JP, Baker SR. Local flaps: cheek and lip reconstruction. JAMA Facial Plast Surg 2013;15:374-82.

3. Holds JB. Lower eyelid reconstruction. Facial Plast Surg Clin North Am 2016;24:183-91.

4. Cha JA, Lee KA. Reconstruction of periorbital defects using a modified Tenzel flap. Arch Craniofac Surg 2020;21:35-40.

5. Chang JW, Lim JH, Lee JH. Reconstruction of midface defects using local flaps: an algorithm for appropriate flap choice. Medicine (Baltimore) 2019;98:e18021.

6. Yoon CS, Kim HB, Kim YK, et al. Relaxed skin tension line-oriented keystone-designed perforator island flaps considering the facial aesthetic unit concept for the coverage of small to moderate facial defects. Medicine (Baltimore) 2019;98:e14167.

7. Hon HH, Chandra SR. Rhomboid flap. Atlas Oral Maxillofac Surg Clin North Am 2020;28:17-22.

8. Kim JH, Jeong HS, Lee BH, et al. Reconstructive modalities according to aesthetic consideration of subunits of the cheek after wide excision of skin cancer. Arch Aesthetic Plast Surg 2016;22:28-34.

9. Bedran EG, Pereira MV, Bernardes TF. Ectropion. Semin Ophthalmol 2010;25:59-65.

10. McKelvie J, Papchenko T, Carroll S, et al. Cicatricial ectropion surgery: a prospective study of long-term symptom control, patient satisfaction and anatomical success. Clin Exp Ophthalmol 2018;46:1002-7.

11. Smith HB, Jyothi SB, Mahroo OA, et al. Patient-reported benefit from oculoplastic surgery. Eye (Lond) 2012;26:1418-23. 
12. Astori IP, Muller MJ, Pegg SP. Cicatricial, postburn ectropion and exposure keratitis. Burns 1998;24:64-7.

13. Cook BE Jr, Bartley GB. Epidemiologic characteristics and clinical course of patients with malignant eyelid tumors in an incidence cohort in Olmsted County, Minnesota. Ophthalmology 1999;106:74650.
14. Shelton ME, Adamson AS. Review and update on evidence-based surgical treatment recommendations for nonmelanoma skin cancer. Dermatol Clin 2019;37:425-33.

15. Orgun D, Hayashi A, Yoshizawa H, et al. Oncoplastic lower eyelid reconstruction analysis. J Craniofac Surg 2019;30:2396-400. 